\title{
Acetábulo de dupla mobilidade na artroplastia total do quadril. A experiência de um hospital universitário
}

\section{Dual mobility cup in total hip arthroplasty. The experiense of the university hospital}

\author{
Raul Frankllim Carvalho Almeida1(1) \\ 1 Grupo de cirurgia do quadril e joelho, Serviço de Ortopedia e \\ Traumatologia, Hospital Universitário, Universidade Federal do \\ Maranhão, São Luís, Maranhão, Brasil \\ Rev Bras Ortop 2020;55(6):708-714.
}

\begin{abstract}
Endereço para correspondência Raul Frankllim Carvalho Almeida, Master, Chefe do Grupo de cirurgia do quadril e joelho, Serviço de Ortopedia e Traumatologia do Hospital Universitário da Universidade Federal do Maranhão (HU-UFMA), Rua Barão de Itapary, 270, 65020-070, Centro, São Luís, Maranhão, Brasil (e-mail: dr.raulalmeida@gmail.com).
\end{abstract}

\section{Resumo \\ Palavras-chave \\ - artroplastia \\ - quadril \\ - prótese de quadril \\ - taça de dupla mobilidade \\ - taxa de luxação \\ - instabilidade articular}

\section{Abstract}

Objetivo Avaliar a incidência de instabilidade protética em uma série consecutiva de 42 casos de artroplastia total do quadril (ATQ) com uso do acetábulo de dupla mobilidade (ADM).

Métodos Estudo retrospectivo com 38 pacientes submetidos a cirurgia de ATQ primária ou de revisão usando o ADM entre janeiro de 2012 e janeiro de 2018. Foram avaliadas as taxas de complicações e de instabilidade após a cirurgia.

Resultados Foram feitas 42 artroplastias em 38 pacientes, com um seguimento mínimo de 16 meses. A média de idade da amostra foi de 60 anos. Utilizamos um ADM não-cimentado em 38 casos e cimentado em 4 casos. Não houve instabilidade precoce ou tardia.

Conclusão A série aqui apresentada prova os bons resultados do ADM em casos de ATQ primaria ou rATQ. A excelente taxa de sobrevivência e a ausência de episódios de instabilidade aumentam a nossa confiança nesse conceito.

Objective To evaluate the incidence of prosthetic instability in a consecutive series of 42 cases of total hip arthroplasty using dual mobility cup (DMC).

Methods A retrospective study of 38 patients undergoing primary or revision total hip arthroplasty (THA) using the acetabular DMC implant between January 2012 and January 2018. The rates of complications and instability after surgery were evaluated. Results In total, 42 arthroplasties were performed in 38 patients, with a minimum follow-up of 16 months. The mean age of the sample was 60 years. In 38 cases, we used a cementless DMC, and, in the other 4 cases, a cemented DMC. There were no cases of early or late instability. recebido

20 de Agosto de 2019

aceito

12 de Dezembro de 2019
DOI https://doi.org/

10.1055/s-0040-1702951. ISSN $0102-3616$.
Copyright $\odot 2020$ by Sociedade Brasileira License terms de Ortopedia e Traumatologia. Published by Thieme Revinter Publicações Ltda, Rio de Janeiro, Brazil 
Keywords

- arthroplasty

- hip

- hip prosthesis

- dual mobility cup

- dislocation rate

- joint instability
Conclusion The present case series proves the good results of the DMC in primary THA and rTHA. The excellent survival rate and the absence of episodes of prosthetic instability increase the confidence in this concept.

\section{Introdução}

A artroplastia total de quadril (ATQ) é uma das intervenções do sistema de saúde de maior sucesso clínico e com melhor custo-benefício do último século. ${ }^{1,2}$ No entanto, apesar da melhora contínua das técnicas cirúrgicas e do desenvolvimento dos implantes, a taxa global de revisão de ATQ (rATQ) permanece elevada, sendo a instabilidade protética a principal causa de revisão deste procedimento em grandes centros de estudo. ${ }^{1,3}$

A instabilidade da ATQ ocorre em aproximadamente $1 \%$ dos casos no primeiro mês de pósoperatório e após 1 ano de cirurgia há um aumento linear do risco de instabilidade, sendo que após 20 anos de pós-operatório o risco cumulativo de instabilidade pode chegar a $7 \% .{ }^{4,5}$ A literatura sugere que mais da metade das luxações ocorrem nos primeiros 3 meses pós-operatório ${ }^{6}$ e mais de $75 \%$ ocorrem no primeiro ano. ${ }^{7}$ A instabilidade após ATQ tem um efeito negativo considerável na qualidade de vida do paciente, especialmente se for recorrente. ${ }^{8}$

Vários fatores foram propostos como influenciadores da estabilidade da ATQ porém muitas questões continuam insolúveis. ${ }^{3,5,9}$ A instabilidade da ATQ pode ser classificada em três grupos de acordo com os fatores de risco, relacionados ao paciente, ao cirurgião e ao tipo de implante utilizado. Dentre os fatores relacionados ao paciente, podemos citar idade avançada, ${ }^{3,7}$ pontuação $\geq 3$ de acordo com a American Society of Anesthesiologists (ASA), ${ }^{9,10}$ gênero feminino, ${ }^{9,11}$ fratura do colo femoral (FCF), ${ }^{3,9}$ osteonecrose da cabeça do fêmur (ONCF), ${ }^{3,9,12}$ displasia do desenvolvimento do quadril (DDQ), ${ }^{9}$ desordens neuromusculares, ${ }^{6,9}$ cirurgia prévia do quadril, ${ }^{9,13}$ e rAQT. ${ }^{5,6}$ Entre os fatores relacionados ao cirurgião estão a orientação dos componentes, ${ }^{11,13}$ a abordagem cirúrgica posterolateral, ${ }^{3,13-15}$ e o volume cirúrgico. ${ }^{7,12,15}$ Entre os fatores intrínsecos ao implante, cita-se o diâmetro da cabeça do fêmur e a razão cabeça-colo ${ }^{3,16}$ (- Tabela 1 ).

Há pouco consenso em relação ao melhor método para prevenir e tratar a instabilidade, particularmente em pacientes de alto risco. ${ }^{4}$

Há mais de 40 anos, Gilles Bousquet e o engenheiro André Rambert ${ }^{19,25}$ tiveram a ideia genial de unificar o conceito de artroplastia de baixa fricção, desenvolvido por Sir John Charnley em $1962,{ }^{17}$ e os benefícios de longevidade das cabeças de grande diâmetro (metal-metal) de MacKee e Watson-Farrar, ${ }^{18}$ gerando maior estabilidade. Baseado nos conceitos supra-citados, o conceito do acetábulo de dupla mobilidade (ADM) surgiu em 1976 na França ${ }^{19}$ (-Fig. 1).

O objetivo deste estudo foi descrever os resultados de uma série de 42 casos de ATQ com uso do ADM com seguimento mínimo de 16 meses.

\section{Materiais e métodos}

Realizamos um estudo retrospectivo com 38 pacientes submetidos a ATQ primária ou rATQ com uso do ADM para prevenção de instabilidade pós-operatória. $O$ estudo incluiu todos os pacientes da instituição que foram submetidos a cirurgia de artroplastia com uso do ADM entre de janeiro de 2012 a janeiro de 2018 . Todos os casos foram operados pelo mesmo cirurgião (RFCA), através da via de acesso pósterolateral sob raquianestesia.

Os dados pré-operatórios dos pacientes foram recolhidos retrospectivamente e prospectivamente nas consultas regulares de seguimento.

Tabela 1 Fatores de risco para instabilidade da ATQ

\begin{tabular}{|l|l|l|}
\hline Paciente & Cirurgião & Implante \\
\hline Idoso $\geq \mathbf{7 5}$ anos & Orientação de componentes & Diâmetro da cabeça femoral \\
\hline Mulher $\geq \mathbf{7 0}$ & Volume cirúrgico & Razão cabeça-colo \\
\hline ASA $\geq 3$ & Abordagem posterolateral & \\
\hline Fratura do colo femoral & & \\
\hline ONCF & & \\
\hline DDQ & & \\
\hline Desordens neuromusculares & & \\
\hline Cirurgia prévia de quadril & & \\
\hline rATQ & & \\
\hline
\end{tabular}




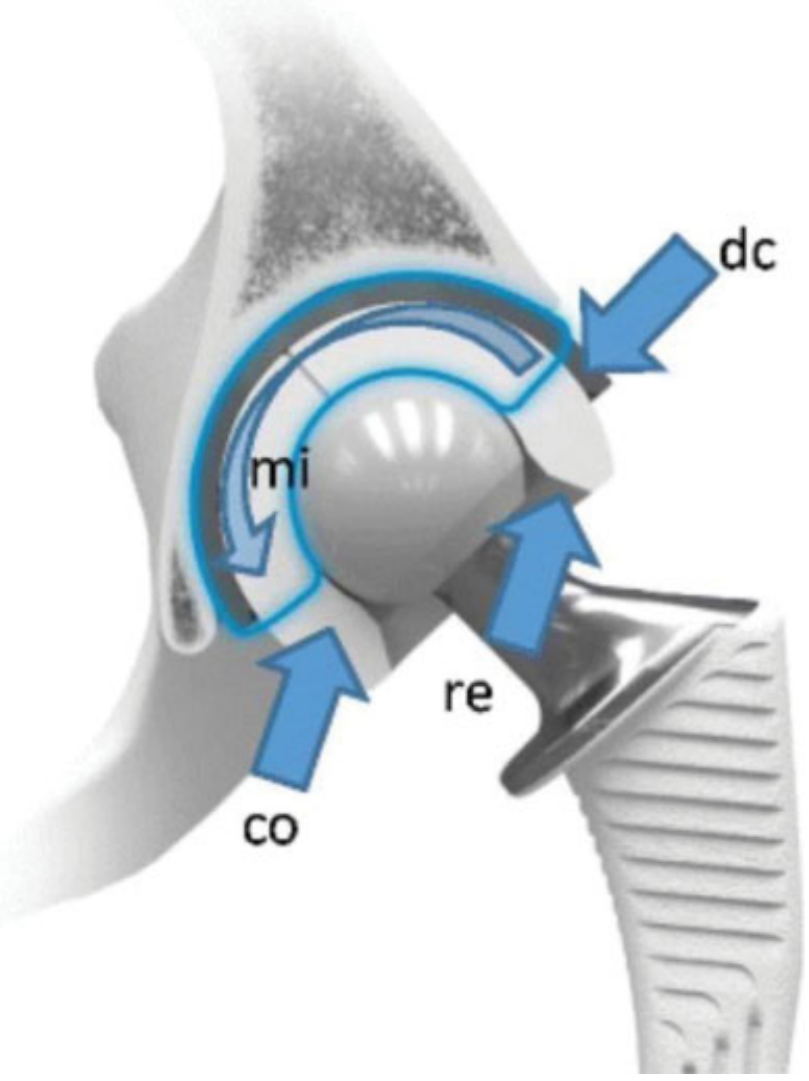

Fig. 1 Sistema de dupla mobilidade: componente acetabular, polietileno móvel e a cabeça femoral. Fonte: https://link.springer.com/article/10.1007/s00264-017-3420-7.

\section{Resultados}

Entre janeiro de 2012 e janeiro de 2018, 42 artroplastias foram feitas em 38 pacientes, com seguimento mínimo de 16 meses. A média de idade da amostra foi de 60 anos (com variação de 17 a 90 anos), sendo 21 (55,5\%) homens e 17 $(44,8 \%)$ mulheres. Por etiologia, houve $14(36,8 \%)$ procedimentos por artrose primária e $28(73,2 \%)$ por causas variadas - 12 por ONCF (2 por abuso de álcool, 2 por FCF, 3 pós- traumáticos, 2 casos de anemia falciforme, 2 casos de uso de corticoide, 1 caso de radioterapia); 4 casos de FCF aguda em idosos; 2 casos de artrite reumatoide; 2 casos de falha de osteosíntese em fratura proximal do fêmur; 2 por pseudoartrose do colo do fêmur; 2 por DDQ; e 1 por epifisiólise femoral proximal (EFP). Também houve 3 rATQ ( 1 caso de instabilidade, 1 caso de afrouxamento asséptico, e 1 caso de conversão de uma hemiartroplastia em ATQ).

Em 38 (90,4\%) casos, usamos ADM não-cimentado (-Fig. 2) e em $4(9,6 \%)$ casos usamos um ADM cimentado (-Fig. 3).

Os dados demográficos pré-operatórios estão listados na - Tabela 2.

A coleta dos dados no pós-operatórios incluiu a lateralidade, o tipo de ATQ realizada (primaria ou revisão), o tipo de ADM utilizado (cimentado ou não-cimentado), a necessidade de pós-operatório na unidade de terapia intensiva (UTI) , uso de hemoderivados, tempo de permanência hospitalar após a cirurgia (em noites) e o seguimento de pós-operatório (-Tabela 3).

Radiografias de controle do pós-operatório imediato foram realizadas para todos os pacientes. No total, foram identificados $2(5,5 \%)$ pacientes com fratura peri-protética estável do femur (Vancouver tipo B1), sendo tratadas de modo conservador e com boa evolução (-Fig. 4).

Outro paciente apresentou paralisia parcial do nervo fibular que evoluiu com resolução completa após 8 semanas de pós-operatório. Não houve nenhum caso de trombose venosa profunda ou de infecção pós-operatórias.

Tivemos um caso de óbito após 2 anos de pós-operatório em um paciente submetido a ATQ por FCF. Ele estava bem da ATQ até a ultima data de avaliação presencial.

\section{Discussão}

A ATQ é um procedimento realizado desde a década de sessenta, e proporcionou melhora significativa na qualidade de vida de pacientes com osteoartrite do quadril. ${ }^{1}$ No entanto, apesar da alta taxa de sucesso, a instabilidade da

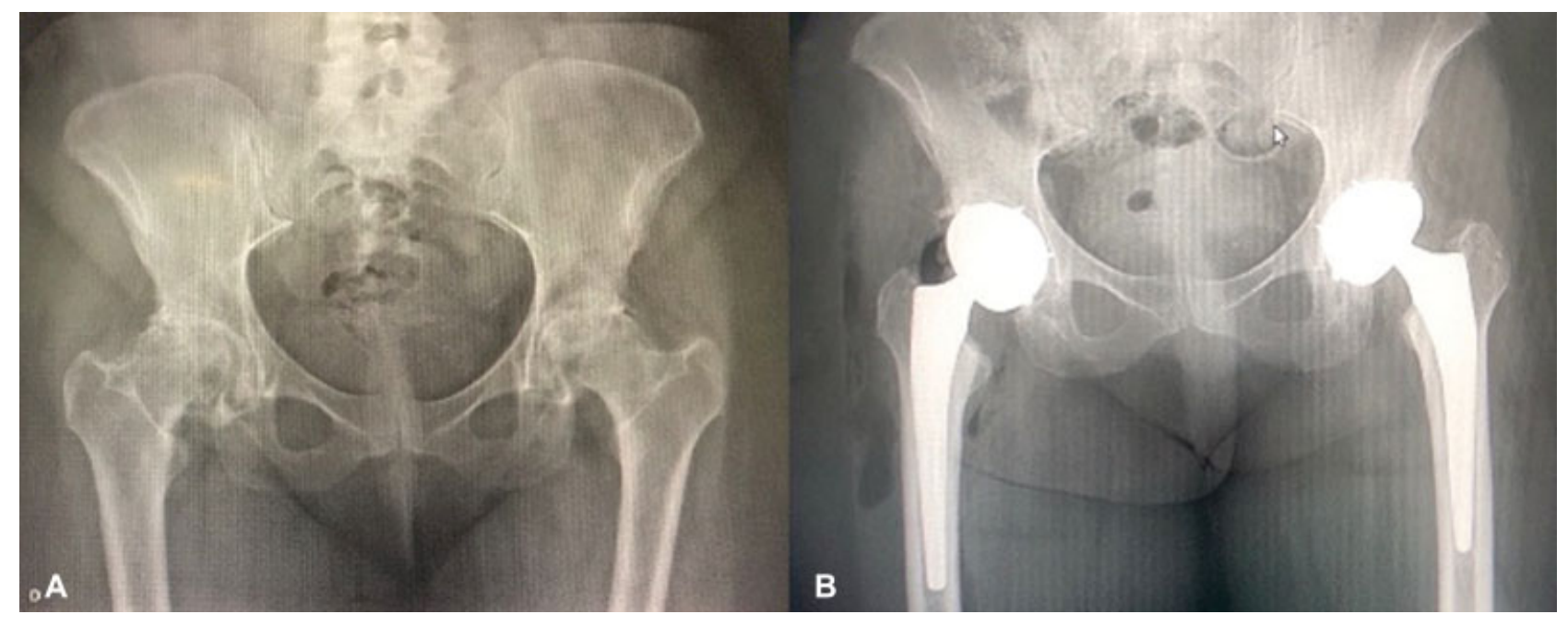

Fig. 2 Radiografia anteroposterior de mulher de 45 anos, com osteonecrose da cabeça femoral (ONCF) bilateral (A), ADM não-cimentado (B). 


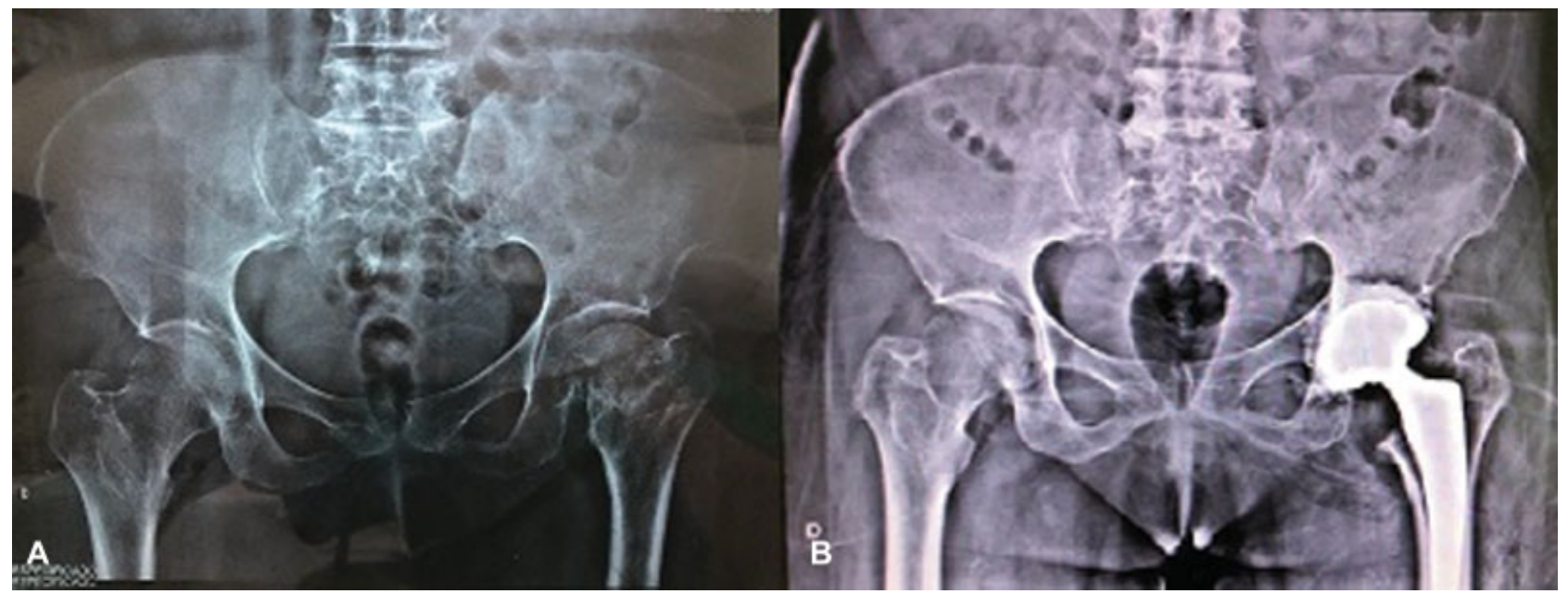

Fig. 3 Radiografia pré-operatória pélvica anteroposterior de mulher de 69 anos, com pseudoartrose do colo femoral (A), e ADM cimentado cimentada no pós-operatório (B).

Tabela 2 Demografia pré-operatória dos pacientes

\begin{tabular}{|l|l|}
\hline Idade & 17-90 anos \\
\hline Gênero & $\begin{array}{l}\text { Masculino: } 21 \\
\text { Feminino: } 17\end{array}$ \\
\hline ASA & $\begin{array}{l}\text { I: } 5 \\
\text { II: } 16 \\
\text { III: } 19\end{array}$ \\
\hline Diagnóstico \\
pré-operatório & $\begin{array}{l}\text { Osteoartrite primária: } 14 \\
\text { Osteonecrose da cabeça do } \\
\text { fêmur: } 12\end{array}$ \\
& $\begin{array}{l}\text { Fratura do colo femoral aguda: } 4 \\
\text { Falha de osteosíntese: } 2 \\
\text { Revisão de artroplastia total } \\
\text { do quadril: } 3\end{array}$ \\
& $\begin{array}{l}\text { Artrite reumatoide: } 2 \\
\text { Pseudoartrose do colo femoral: } 1 \\
\text { Displasia do desenvolvimento } \\
\text { do quadril: } 1\end{array}$ \\
& Epifisiólise femoral proximal: 1 \\
\hline
\end{tabular}

Tabela 3 Dados pós-operatórios

\begin{tabular}{|l|l|}
\hline Lateralidade & $\begin{array}{l}\text { Direito 15 } \\
\text { Esquerdo 21 } \\
\text { Bilateral 3 }\end{array}$ \\
\hline ADM & $\begin{array}{l}\text { Cimentada: 4 } \\
\text { Não-cimentada: 38 }\end{array}$ \\
\hline ATQ & $\begin{array}{l}\text { Primária: 39 } \\
\text { Revisão: 3 }\end{array}$ \\
\hline Transfusão sanguínea & $\begin{array}{l}\text { Sim: } 19 \\
\text { Não: } 23\end{array}$ \\
\hline UTI & $\begin{array}{l}\text { Sim: } 20 \\
\text { Não: 22 }\end{array}$ \\
\hline TPH (noites) & 4 \\
\hline Seguimento médio & 16 (12-74) meses \\
\hline
\end{tabular}

ATQ continua sendo um problema de difícil manejo para o paciente e para o cirurgião. ${ }^{2-5}$

Bozic et al. $^{4}$ avaliaram as causas de falha em 51.345 procedimentos de rATQ feitos nos Estados Unidos no período entre $1^{\circ}$ de outubro de 2005 e 31 de dezembro de 2006. A causa mais comum de rATQ foi instabilidade (22,5\%). Revisões por luxação totalizaram $9 \%$ de todas as revisões de ATQ primária no Swedish Hip Arthroplasty Register. É a segunda razão mais comum para revisão. O Australian National Joint Replacement Registry relatou uma proporção de $26 \%$ de rATQ por luxação em 2011. ${ }^{3}$ Em contraste com a literatura, a incidência de instabilidade da ATQ na França é a quinta causa de rATQ. ${ }^{9,20,21}$

A luxação pós-ATQ é multifatorial (-Tabela 1). Os fatores relacionados ao paciente não mudam. No entanto, os fatores relacionados à escolha da abordagem cirúrgica e ao tipo de implante cabem ao cirurgião. A prevenção da instabilidade após a cirurgia de ATQ é a melhor estrategia. Na última década, houve um aumento exponencial de artigos publicados sobre o uso do ADM na ATQ. Muitos desses artigos mostram taxas muito baixas de luxação, ou até a ausência dessa complicação. ${ }^{3,5,9,19-24}$ O sistema de dupla mobilidade foi criado na França em 1976 para aumentar a estabilidade e melhorar a mobilidade da articulação com baixa fricção. ${ }^{19}$

Em nossa instituição, usamos o ADM desde janeiro de 2012. Inicialmente, foi indicado somente em pacientes idosos com FCF e em pacientes com alto risco de instabilidade após ATQ. No entanto, à medida que fomos nos familiarizando com a técnica e nos aprofundando no conhecimento em biomecânica e nos princípios do sistema de dupla mobilidade, as indicações foram estendidas gradualmente. Atualmente, recomendamos o ADM para pacientes de qualquer idade e diagnóstico, posto que não observamos qualquer problema no seguimento. A ausência de instabilidade foi confirmada pelos resultados da literatura. ${ }^{16,24}$

Para Woo e Morrey, ${ }^{14}$ o risco de luxação é duas vezes maior para mulheres em comparação com homens. Coventry ${ }^{11}$ observou que a taxa de luxação da ATQ foi 3 vezes maior 


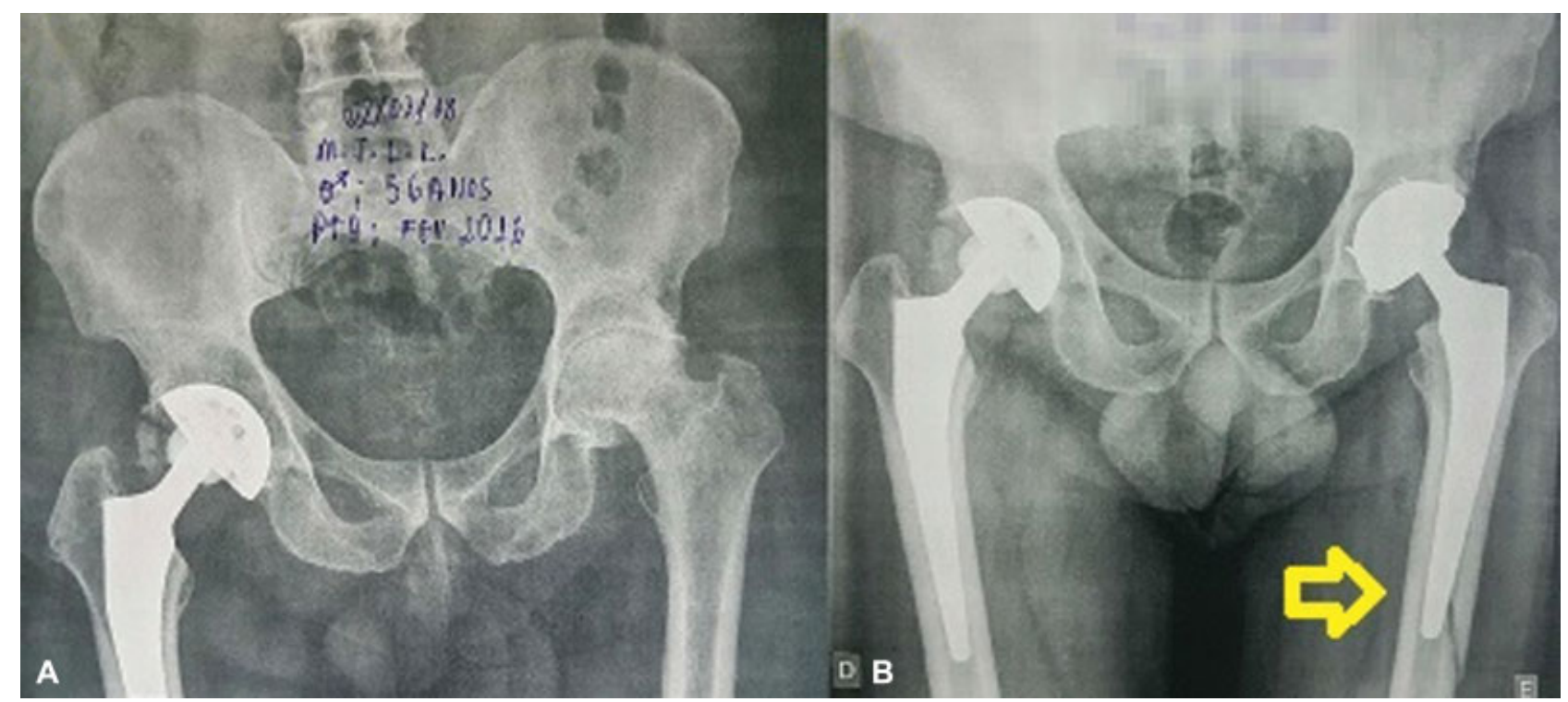

Fig. 4 Radiografia pré-operatória pélvica anteroposterior de homem de 56 anos, com ONCF esquerda (A), e radiografia pós-operatória de ADM não-cimentado com fratura de fêmur periprostética estável, Vancouver tipo B1 (B). As fraturas foram tratadas de forma conservadora e com sucesso.

para mulheres em comparação com homens 5 anos após a cirurgia. Para Bozic et al., ${ }^{4}$ procedimentos de revisão de ATQ eram mais comumente relatados na faixa etária de 75 a 84 anos (27,0\%). Para Guyen et al., ${ }^{9}$ somente o gênero não é o suficiente para indicar o uso sistemático do ADM. Eles consideram o gênero feminino um fator de risco para luxação quando associado à idade de 70 anos ou mais. No presente estudo, havia 17 mulheres. 0 escore da ASA foi identificado como um bom risco para a variável de luxação porque está relacionado a co-morbidades que aumentam com a idade e complicam a recuperação muscular no pós-operatório. Os autores observaram que a pontuação $\mathrm{ASA} \geq 3$ representava um risco significativo para luxação. ${ }^{10}$ Nesse estudo, $42.8 \%$ (19) dos pacientes tinham um escore $A S A \geq 3$. Todos os pacientes tiveram boa evolução.

No período entre 1968 e 1978, foram realizadas 10.500 cirurgias de ATQ na Mayo Clinic. Analises multivariadas foram realizadas para investigar os fatores de risco para instabilidade. A taxa de instabilidade geral foi de 3,2\% (331 casos). O fator de risco mais forte para instabilidade após ATQ foi a presença de cirurgia prévia no quadril operado com taxa de $4,8 \%$. Nos casos sem cirurgia prévia a taxa de instabilidade foi de $2,4 \%$. A via de acesso de acesso antero-lateral apresentou uma taxa de instabilidade de $2,3 \%$, enquanto a via póstero-lateral foi de 5,8\%. 0 tamanho da cabeca femoral não influenciou a taxa de instabilidade. ${ }^{14}$ Berry et al., analisaram 21.047 cirurgias de ATQ com enfase nos fatores de risco para instabilidade. Os pacientes foram acompanhados regularmente e avaliados no pós-operatório de 1 ano, 5 anos, 10 anos e 20 anos. Foram observados 868 casos de instabilidade $(4,12 \%)$. Analise multivariada dos dados mostrou que a cabeça femoral de maior diâmetro foi fator de proteção contra instabilidade em todas as vias de acesso utilizada. A via de acesso póstero-lateral apresentou o dobro do risco de instabilidade $(6,9 \%)$ quando comparada com a via trans- trocanterica (3,4 \%) e antero-lateral (3,1\%). A pior combinação foi observada na via de acesso postero-lateral com cabeca femoral de $22 \mathrm{~mm}(12,1 \%) .{ }^{16}$ Bouchet et al. (2011), analisaram duas séries de casos de artroplastia primaria (213 cirurgias) operadas pelo mesmo cirurgião usando a via de acesso póstero-lateral com o objetivo da avaliar a taxa de instabilidade. No primeiro grupo (2003-2005) foram incluídos 108 pacientes submetidos a ATQ por via convencional. No grupo controle ( 2005-2007), 105 pacientes foram operados com uso do ADM. Todos os implantes utilizaram cabeça $28 \mathrm{~mm}$. Foram comparados idade, sexo, peso, diagnostico, tipo de anestesia e o escore ASA. $O$ seguimento minimo foi de 1 ano. No grupo convencional a taxa de instabilidade foi de $4,63 \%$ ( 5 casos ). No grupo dupla mobilidade, não houve nenhum episódio de instabilidade. ${ }^{20}$

Outro grupo com risco aumentado para instabilidade da ATQ é formado por pacientes com histórico de cirurgia prévia do quadril. ${ }^{9,12,14}$ Nos casos de ATQ em situações complexas, a taxa de luxação pode variar de 5 a $30 \%$, em decorrência da perda óssea e insuficiência de músculos e tecidos do quadril. ${ }^{16} \mathrm{O}$ uso de ADM em ATQ complexa mostrou taxa de deslocamento de $1 \%$ a $10 \%$ em seguimento de 8 anos. ${ }^{25}$ No presente estudo, houve $7(16,6 \%)$ casos de ATQ em pacientes com historia de cirurgia prévia no quadril, incluindo $2(5,5 \%)$ casos de falha de osteossíntese em fratura do femur proximal. Em todos os casos houve boa evolução.

A incidência relatada de luxação após rATQ pode variar de 22,5 a $39 \%,{ }^{4-6}$ sendo que em alguns casos, uma segunda revisão cirúrgica é um procedimento inevitável, particularmente em pacientes com múltiplos fatores de risco para instabilidade. A melhor estratégia é adequar a escolha da abordagem cirúrgica e os implantes para prevenir problemas futuros. ${ }^{5}$ No presente estudo, tivemos apenas um caso de rATQ por instabilidade. No entanto, a paciente apresentava vários fatores de risco associados, evoluindo com instabilidade após o novo procedimento 
de rATQ. Paciente do sexo feminino, portadora de artrite reumatoide, usando corticosteroides, histórico de abuso de álcool, operada previamente três vezes e com baixa reserva muscular e óssea. Um acetábulo de dupla mobilidade foi cimentado no interior da cúpula acetabular prévia que encontrava-se firme. Esta é uma vantagem adicional do sistema de dupla mobilidade: versatilidade. A paciente teve uma boa evolução, sem instabilidade.

Em muitos estudos, o diagnóstico de fratura aguda do colo do femur está associado ao risco aumentado de rATQ por instabilidade. 3,9,12 Lu-Yao et al. encontraram uma taxa de instabilidade de $10,7 \%$ em pacientes com fratura aguda do colo do femur tratados com ATQ convencional. ${ }^{26}$ Tarasevicius et al. ${ }^{23}$ compararam ATQ de dupla mobilidade com ATQ convencional em pacientes com fratura aguda do colo do femur. Depois de 1 ano de seguimento de pós-operatório, nenhuma luxação foi relatada no grupo com ADM. No grupo de ATQ convencional foarm encontrados 8 (14,3\%) casos. Em um estudo prospectivo e multicêntrico realizado na Franca (2012), Adam et al. avaliaram 214 pacientes portadores de fratura aguda do colo do femur submetidos a tratamento cirúrgico com ATQ usando o ADM. A média de idade foi de 83 anos (70-103 anos). Observaram $3(1,4 \%$ ) casos de luxação no pós-operatório que foram submetidos a redução incruenta com sucesso. Não houve recorrência de luxação nesta serie. $^{27}$

Apesar da literatura médica apresentar fortes evidências sobre a segurança do sistema de dupla mobilidade na prevenção da instabilidade após a ATQ ainda observamos dois grandes pontos de questionamento. Primeiro temos a instabilidade intra-protética do sistema de dupla mobilidade. Outro ponto de forte questionamento é a sobrevida do implante em pacientes jovens. A luxação intra-protética é uma complicação específica do sistema de dupla mobilidade, induzida pela perda da borda de retenção de polietileno, resultando na separação da cabeça femoral da cobertura de polietileno. No entanto, artigos mostram que essa complicação ocorreu na primeira geração de implantes em que foram utilizados polietilenos convencionais com cabeças de $22 \mathrm{~mm}$. As taxas de luxação intra-protética com seguimento médio de 4 a 17 anos, variam de 1,9\% a 5,2\% de acordo com o modelo e a geração do implante utilizado. ${ }^{9,19,28}$ Sobre a sobrevivência do implante em pacientes mais jovens, há vários estudos mostrando taxas de sobrevivência similares às dos implantes convencionais. 3,5,19,20,22,24,25,27 Não houve casos de luxação na série do presente estudo.

O poder estatístico deste estudo certamente é limitado pelo tamanho pequeno da amostra.

\section{Conclusão}

A série aqui apresentada prova os bons resultados do ADM em casos de ATQ primaria ou rATQ. A excelente taxa de sobrevivência e a ausência de episódios de instabilidade aumentam a nossa confiança nesse conceito.

Conflito de interesses

Os autores declaram não haver conflito de interesses.

\section{Referências}

1 Learmonth ID, Young C, Rorabeck C. The operation of the century: total hip replacement. Lancet 2007;370(9597):1508-1519

2 Kurtz S, Ong K, Lau E, Mowat F, Halpern M. Projections of primary and revision hip and knee arthroplasty in the United States from 2005 to 2030. J Bone Joint Surg Am 2007;89(04):780-785

3 Hailer NP, Weiss RJ, Stark A, Kärrholm J. The risk of revision due to dislocation after total hip arthroplasty depends on surgical approach, femoral head size, sex, and primary diagnosis. An analysis of 78,098 operations in the Swedish Hip Arthroplasty Register. Acta Orthop 2012;83(05):442-448

4 Bozic KJ, Kurtz SM, Lau E, Ong K, Vail TP, Berry DJ. The epidemiology of revision total hip arthroplasty in the United States. J Bone Joint Surg Am 2009;91(01):128-133

5 Faldini C, Stefanini N, Fenga D, et al. How to prevent dislocation after revision total hip arthroplasty: a systematic review of the risk factors and a focus on treatment options. J Orthop Traumatol 2018;19(01):17

6 Daly PJ, Morrey BF. Operative correction of an unstable total hip arthroplasty. J Bone Joint Surg Am 1992;74(09):1334-1343

7 Meek RM, Allan DB, McPhillips G, Kerr L, Howie CR. Epidemiology of dislocation after total hip arthroplasty. Clin Orthop Relat Res 2006;447(447):9-18

8 Enocson A, Pettersson H, Ponzer S, Törnkvist H, Dalén N, Tidermark J. Quality of life after dislocation of hip arthroplasty: a prospective cohort study on 319 patients with femoral neck fractures with a one-year follow-up. Qual Life Res 2009;18(09):1177-1184

9 Guyen O, Chen QS, Bejui-Hugues J, Berry DJ, An KN. Unconstrained tripolar hip implants: effect on hip stability. Clin Orthop Relat Res 2007;455(455):202-208

10 Jolles BM, Zangger P, Leyvraz PF. Factors predisposing to dislocation after primary total hip arthroplasty: a multivariate analysis. J Arthroplasty 2002;17(03):282-288

11 Coventry MB. Late dislocations in patients with Charnley total hip arthroplasty. J Bone Joint Surg Am 1985;67(06):832-841

12 Bourne RB, Mehin R. The dislocating hip: what to do, what to do. J Arthroplasty 2004;19(04, Suppl 1):111-114

13 Biedermann R, Tonin A, Krismer M, Rachbauer F, Eibl G, Stöckl B. Reducing the risk of dislocation after total hip arthroplasty: the effect of orientation of the acetabular component. J Bone Joint Surg Br 2005;87(06):762-769

14 Woo RY, Morrey BF. Dislocations after total hip arthroplasty. J Bone Joint Surg Am 1982;64(09):1295-1306

15 Sanchez-Sotelo J, Haidukewych GJ, Boberg CJ. Hospital cost of dislocation after primary total hip arthroplasty. J Bone Joint Surg Am 2006;88(02):290-294

16 Berry DJ, von Knoch M, Schleck CD, Harmsen WS. Effect of femoral head diameter and operative approach on risk of dislocation after primary total hip arthroplasty. J Bone Joint Surg Am 2005;87(11): 2456-2463

17 Charnley J. The long-term results of low-friction arthroplasty of the hip performed as a primary intervention. J Bone Joint Surg $\mathrm{Br}$ 1972;54(01):61-76

18 McKee GK, Watson-Farrar J. Replacement of arthritic hips by the McKee-Farrar prosthesis. J Bone Joint Surg Br 1966;48(02): 245-259

19 Chughtai M, Mistry JB, Diedrich AM, et al. Low Frequency of Early Complications With Dual-mobility Acetabular Cups in Cementless Primary THA. Clin Orthop Relat Res 2016;474(10): 2181-2187

20 Bouchet R, Mercier N, Saragaglia D. Posterior approach and dislocation rate: a 213 total hip replacements case-control study comparing the dual mobility cup with a conventional $28-\mathrm{mm}$ metal head/polyethylene prosthesis. Orthop Traumatol Surg Res 2011;97(01):2-7

21 Combes A, Migaud H, Girard J, Duhamel A, Fessy MH. Low rate of dislocation of dual-mobility cups in primary total hip arthroplasty. Clin Orthop Relat Res 2013;471(12):3891-3900 


\section{Acetábulo de dupla mobilidade na artroplastia total do quadril Almeida}

22 Prudhon JL, Ferreira A, Verdier R. Dual mobility cup: dislocation rate and survivorship at ten years of follow-up. Int Orthop 2013; 37(12):2345-2350

23 Tarasevicius S, Robertsson O, Dobozinskas P, Wingstrand H. A comparison of outcomes and dislocation rates using dual articulation cups and THA for intracapsular femoral neck fractures. Hip Int 2013;23(01):22-26

24 Darrith B, Courtney PM, Della Valle CJ. Outcomes of dual mobility components in total hip arthroplasty: a systematic review of the literature. Bone Joint J 2018;100-B(01):11-19

25 De Martino I, Triantafyllopoulos GK, Sculco PK, SculcoTP. Dual mobility cups in total hip arthroplasty. World J Orthop 2014;5(03):180-187

26 Lu-Yao GL, Keller RB, Littenberg B, Wennberg JE. Outcomes after displaced fractures of the femoral neck. A meta-analysis of one hundred and six published reports. J Bone Joint Surg Am 1994;76 (01):15-25

27 Adam P, Philippe R, Ehlinger M, et al. French Society of Orthopaedic Surgery and Traumatology (SoFCOT). Dual mobility cups hip arthroplasty as a treatment for displaced fracture of the femoral neck in the elderly. A prospective, systematic, multicenter study with specific focus on postoperative dislocation. Orthop Traumatol Surg Res 2012;98(03):296-300

28 Philippot R, Boyer B, Farizon F. Intraprosthetic dislocation: a specific complication of the dual-mobility system. Clin Orthop Relat Res 2013;471(03):965-970

29 Vielpeau C, Lebel B, Ardouin L, Burdin G, Lautridou C. The dual mobility socket concept: experience with 668 cases. Int Orthop 2011;35(02):225-230 